\title{
Collective motions in globally coupled tent maps with stochastic updating
}

\author{
Satoru Morita* \\ Department of Systems Engineering, Shizuoka University, 3-5-1 Jouhoku, Hamamatsu 432-8561, Japan \\ Tsuyoshi Chawanya \\ Department of Mathematics, Graduate School of Science, Osaka University, Toyonaka 560-0043, Japan
}

(Received 13 September 2001; published 15 March 2002)

We study a generalization of globally coupled maps, where the elements are updated with probability $p$. When $p$ is below a threshold $p_{c}$, the collective motion vanishes and the system is the stationary state in the large size limit. We present the linear stability analysis.

DOI: 10.1103/PhysRevE.65.046201

PACS number(s): 05.45.Jn, 05.70.Ln, 82.40.Bj

\section{INTRODUCTION}

The globally coupled maps (GCM) are introduced as a simple model capturing the essential features of nonlinear dynamical systems with many degrees of freedom [1]. One of the most interesting phenomena seen in such systems is the emergence of the collective motion [2-4]. The collective motion is characterized by a time dependence of the macroscopic variable in the large size limit [5-16]. In this paper, we consider a variation of GCM to include asynchronous updating.

The general form of GCM is written in the following way:

$$
x_{t+1}(i)=f\left(x_{t}(i)\right)+\frac{K}{N} \sum_{i^{\prime}=1}^{N} g\left(x_{t}\left(i^{\prime}\right)\right),
$$

where $t$ represents discrete time steps, $i$ specifies each element, $K$ gives the coupling strength, and $N$ is the system size. All elements are updated synchronously in the deterministic way through the mean field

$$
h_{t} \equiv \frac{1}{N} \sum_{i=1}^{N} g\left(x_{t}(i)\right) .
$$

Since the collective motions have been studied analytically in globally coupled tent maps [5-13], we specifically consider tent maps as follows:

$$
\begin{gathered}
f(x) \equiv a\left(\frac{1}{2}-|x|\right), \\
g(x) \equiv f(x)-\bar{f} .
\end{gathered}
$$

Here $\bar{f}$ is a constant determined from the average of $f(x)$ over the natural invariant measure of the map $x \mapsto f(x)$, i.e.,

$$
\bar{f}=\int f(x) \rho_{*}(x) d x,
$$

where $\rho_{*}(x)$ represents the natural invariant density. By the above choice of $g(x), h_{t}=0$ is a stationary solution for the

\footnotetext{
*Electronic address: morita@sys.eng.shizuoka.ac.jp
}

large size limit $(N \rightarrow \infty)$ of Eq. (1), and the corresponding stationary distribution is proportional to $\rho_{*}(x)$. Equation (3) looks a little different from well-known form of GCM system

$$
x_{t+1}(i)=(1-\epsilon) f\left(x_{t}(i)\right)+\frac{\epsilon}{N} \sum_{i} f\left(x_{t}(i)\right),
$$

which is obtained as a mean-field approximation for the coupled map lattice with diffusion coupling. In the case of the tent map system, however, the diffusion form (5) is scaled into Eq. (1) [12].

The collective motions in GCM (1) with Eq. (3) are classified into two types according to the gradient $a$ of the tent map. First, in the case of $a<1$, the synchronized chaos is stable. In this case, the map $x \mapsto f(x)$ has the stable fixed point

$$
x_{*}=\frac{a}{2 a+2} .
$$

Thus, $\rho_{*}(x)=\delta\left(x-x_{*}\right)$, i.e., $g(x)=f(x)-f\left(x_{*}\right)$. Since the gradient of $f(x)$ is smaller than 1 , the difference between any pair of elements diminishes. Thus all the elements behave identically after some initial transient. Here we concentrate on the long-term behavior and assume the system is onecluster state. Then temporal evolution for $x_{t}$ and $h_{t}$ is obtained as follows:

$$
\begin{aligned}
x_{t+1} & =\left[\frac{1}{2}+\frac{a K}{2+2 a}-(1+K)\left|x_{t}\right|\right], \\
h_{t+1} & =a\left[x_{*}-\left|x_{*}+(1+K) h_{t}\right|\right] .
\end{aligned}
$$

When $K$ is so large that $a(1+K)>1$, the fixed point $h_{t}=0$ is unstable. Then, the motion of the mean field is onedimensional chaos, which obeys Eq. (7).

Second, in the case of $a>1$, all elements are fully desynchronized and behave as if they are mutually independent. Nevertheless, the fluctuation of the mean field dose not vanish in the large size limit [2]. Thus, the system has a nontrivial collective motion [5].

From a realistic viewpoint, however, the synchronous updating is not always plausible as models of real systems 


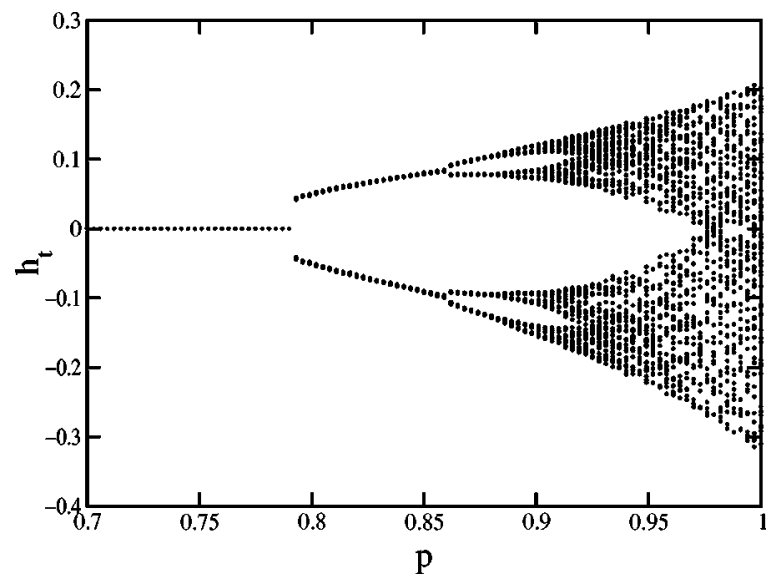

FIG. 1. Bifurcation diagram for evolution of the mean field as a function of the updating rate $p$ in the case of $a=0.9, K=0.7, N$ $=10^{5}$. For given values of $p$, the value of the mean field is plotted for 64 successive steps after $10^{4}$ steps as transient.

[17-22]. In some cases, for example, neural networks, an independent choice of the times at which the state of a given element is updated should provide a better approximation. Abramson and Zanette have numerically found that, for globally coupled logistic map with completely stochastic updating, the fluctuation of the mean field can vanish in the large size limit [19]. In this paper, we study the stochastic updating model as follows:

$$
x_{t+1}(i)= \begin{cases}f\left(x_{t}(i)\right)+K h_{t} & \text { with probability } p, \\ x_{t}(i) & \text { with probability }(1-p) .\end{cases}
$$

Here the definition of the mean field (2) is not changed. At each time step, update the elements with probability $p$ satisfying $0<p \leqslant 1$. When the updating rate $p$ is equal to 1 , the model (8) becomes the synchronous updating model (1). On the other hand, when $p$ decreases to 0 , the model (8) approaches the completely asynchronous updating model [25]. Thus, the value of $(1-p)$ represents the strength of the asynchronousnism. The purpose of this paper is to investigate how the collective behavior changes when the updating probability $p$ varies, mainly by the linear stability analysis of the stationary state in the large size limit.

There is another extension of GCM to include sequential updating [22]. In this case, the state of the elements is updated according to a given sequence that is fixed during the evolution. Jiang et al. showed numerically that the sequential updating induces a variety of collective behaviors such as spatial bifurcation cascades and traveling wave. In contrast to the stochastic updating, this type of asynchronousnism does not reduce the collective motion. In this paper, we concentrate on the stochastic updating model. One reason is that we are interested in the situation with almost synchronous updating, where a portion of elements are not updated by accident. Another reason is that the stochastic updating model can be treated analytically with using the distribution function.
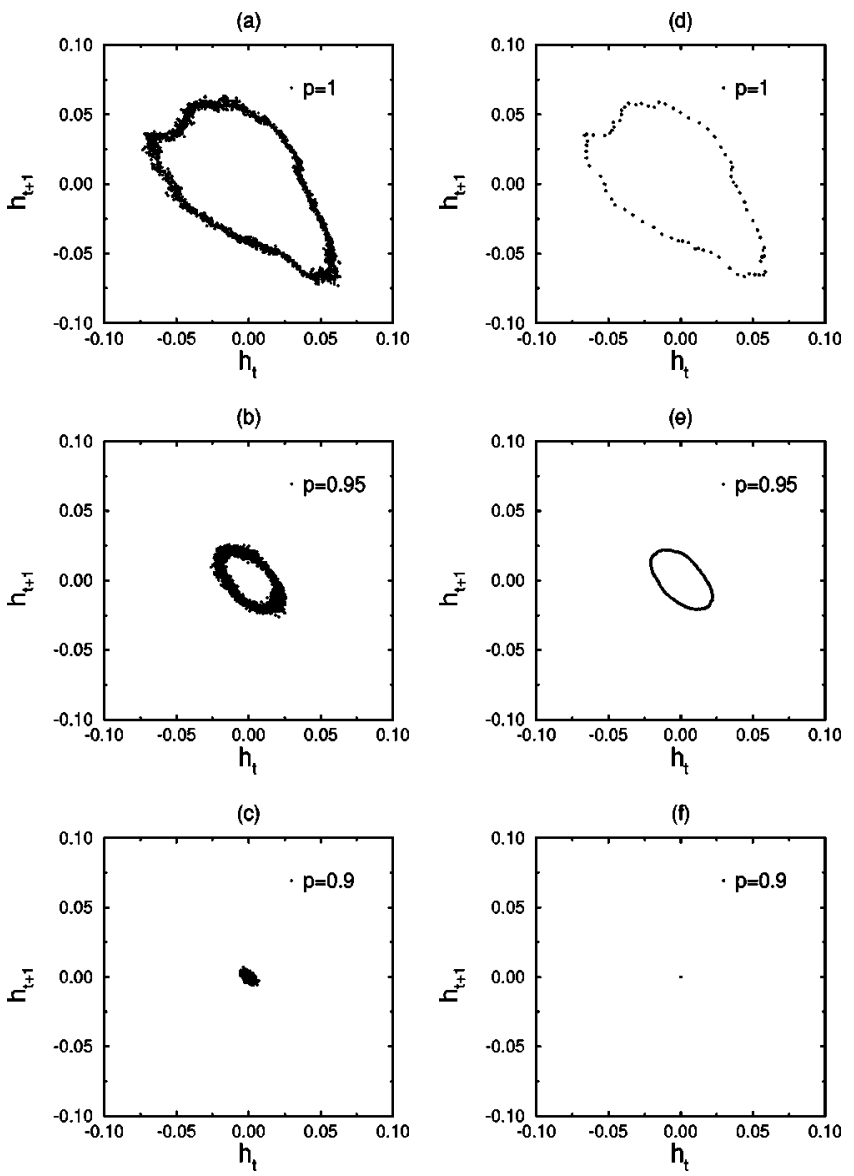

FIG. 2. Examples of the return maps of the mean field are shown ( $a=1.625, K=0.4)$. While (a), (b), and (c) are obtained by the direct numerical calculation of Eq. (8) with the size of $N$ $=10^{5}$, (d), (e), and (f) are obtained by the numerical calculation of Eq. (11) with $2^{18}$ grids. (a) and (d) are for $p=1$, (b) and (e) are for $p=0.95$, (c) and (f) are for $p=0.9$. We plot 2000 points after $10^{4}$ steps as transient.

\section{NUMERICAL RESULTS}

In this section, we present the numerical results for the model (8). First, we examine the case of $a<1$. When $p=1$, the synchronized chaos is observed. On the other hand, when $p<1$, some elements are updated and the others are fixed at each time step. Hence, even when a pair of elements have the same value at a moment, they can have different values at the next time. As a result, the synchronized chaos is broken. When $p$ is near 1 , the synchronized state is blurred slightly. As $p$ decreases, a sequence of bifurcations is seen (in Fig. 1). It resembles the period doubling cascade. However, there is a finite jump at this bifurcation in contrast of the usual period doubling bifurcation. This discontinuity is due to the fact that the map $f(x)$ is piecewise linear. When $p$ is smaller than a threshold value $p_{c}$, all elements fall into the fixed point (6) and the mean field $h_{t}$ becomes 0 . Thus, the collective motion vanishes below the threshold $p_{c}$.

Second, we examine the case of $a>1$, where the nontrivial collective motion is seen for $p=1$. Figures 2(a), 2(b), and 2(c) show the motions of the mean field for some values of $p$, with $a=1.625, K=0.4$, and $N=10^{5}$. The amplitude of 
motion of the mean field decreases as $p$ decreases.

It should be noted that the dynamics of each element is not deterministic due to the updating rule. Thus, the meanfield value is blurred if the system size is finite. Even for $p$ $=1$, the finite size effect works on the motion of the mean field as an internal noise [5]. For that reason, it is useful to consider the large size limit. In the large size limit, the ensemble of the elements is characterized by its distribution. In the synchronous updating case, the evolution of the distribution function $\rho_{t}(x)$ obeys the nonlinear Frobenius-Perron equation

$$
\left.\rho_{t+1}(x)=\mathcal{F} \rho_{t}(x) ; h_{t}\right] \equiv \frac{\rho_{t}\left(y_{+}\right)+\rho_{t}\left(y_{-}\right)}{a},
$$

where $\mathcal{F}$ represents the Frobenius-Perron operator, and $y_{+}$ and $y_{-}$are the two preimage of $x$, i.e., $x=f\left(y_{ \pm}\right)+K h_{t}$. Here the mean field is determined in the integral form,

$$
h_{t}=\int g(x) \rho_{t}(x) d x
$$

In the stochastic updating case $(p<1)$, the evolution of $\rho_{t}(x)$ is described as

$$
\rho_{t+1}(x)=p \mathcal{F}\left[\rho_{t}(x) ; h_{t}\right]+(1-p) \rho_{t}(x) .
$$

Despite the stochastic updating, the distribution function evolves in the deterministic way.

In order to calculate Eq. (11) numerically, we approximate the distribution function $\rho_{t}(x)$ by dividing the relevant interval of $x$ into $m$ small intervals. The evolution of the distribution is described by the $m \times m$ transfer matrix that depends on time through the mean field $h_{t}$. Here we construct the transfer matrix by applying the method by Binder and Campos [23]. Figures 2(d), 2(e), and 2(f) show the motion of the mean field calculated by this method with the parameter values corresponding to Figs. 2(a), 2(b), and 2(c), respectively. The direct calculations of Eq. (8) compare successfully with the results of Eq. (11), except for the fluctuation due to the finite size effect. As is seen from Fig. 2(f), when $p$ is smaller than a threshold value $p_{c}$, the collective motion vanishes like the case of $a<1$. In the stationary state, all elements are still scattered and behave chaotically in contrast to the case of $a<1$. The fluctuation of the mean field resides for finite size systems [Fig. 2(c)]. Note that when the mean field vanishes, all elements are independent of each other, because the coupling term becomes 0 . The amplitude of the mean field tends to decrease as asynchronousnism grows. This suggests that asynchronous updating weakens the coherence among elements. On the other hand, the frequency of the collective motion is proportional to the updating rate $p$. This can be explained by the fact that the time scale is given by $1 / p$.

\section{LINEAR STABILITY ANALYSIS OF THE STATIONARY STATES}

The result of numerical simulation indicates that there exists a threshold value $p_{c}$ for updating rate. It is observed that the collective motion vanishes, and the stationary state [with

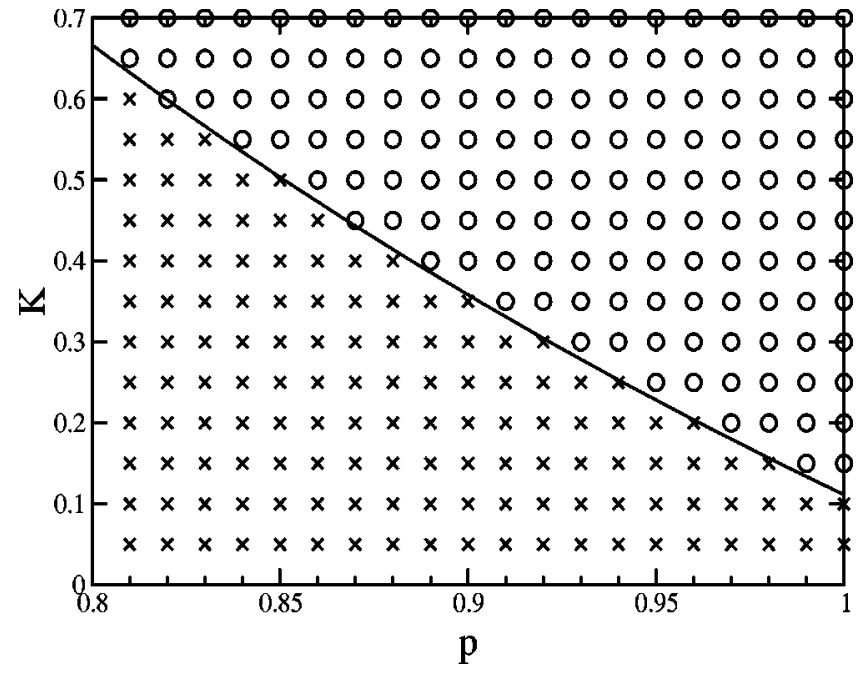

FIG. 3. Phase diagram of the collective motion for $a=0.9$. The numerical results are obtained for $N=10^{4}$. Circles and crosses represent the cases of $\left\langle h^{2}\right\rangle^{1 / 2}>10^{-6}$ and $\left\langle h^{2}\right\rangle^{1 / 2}<10^{-6}$, respectively. Here the angular bracket denotes the average value of the enclosed quantity over 2000 time steps. The solid line represents the theoretical prediction (14).

distribution $\left.\rho_{t}(x)=\rho_{*}(x)\right]$ is realized for $p$ smaller than $p_{c}$. In this section, we present the linear stability analysis of the stationary state to estimate the value of $p_{c}$.

First, we consider the case of $a<1$. This case is simpler, because all elements have the identical fixed value $x_{*}(>0)$ in the stationary state. Considering a small perturbation from $x_{*}$, we assume that every element has a positive value. Since $\rho_{t}(x)$ for $x<0$ is 0 , the evolution of $\rho_{t}(x)$ is rewritten as

$$
\rho_{t+1}(x)=\frac{p}{a} \rho_{t}\left(\frac{a-2 x+2 K h_{t}}{2 a}\right)+(1-p) \rho_{t}(x) .
$$

From Eqs. (10) and (12), we obtain the dynamics of the mean field obeys

$$
h_{t+1}=(-a p-a p K+1-p) h_{t} .
$$

Thus, the stationary state is stable when $\mid-a p-a p K+1$ $-p \mid<1$. Consequently, the threshold value $p_{c}$ is estimated as

$$
p_{c}=\frac{2}{a(1+K)+1} \text {. }
$$

In the case of Fig. $1, p_{c}=0.790 \ldots$. The theoretical prediction agrees well with the numerical simulation (see also Fig. 3).

Second, we consider the case of $a>1$. The stationary distribution function $\rho_{*}(x)$ is expanded into series of step functions as follows $[9,12]$ :

$$
\rho_{*}(x)=\sum_{k=1} C_{k} \theta\left(x-X_{k}\right)
$$




$$
\begin{gathered}
X_{k} \equiv f^{k}(0), \\
C_{k} \equiv C_{1}\left\{\left(f^{k-1}\right)^{\prime}[f(0)]\right\}^{-1},
\end{gathered}
$$

where $\theta(x)$ is a step function: 1 for $x \geqslant 0$ and 0 for $x<0$. Thus, $X_{k}$ and $C_{k}$ represent the locations and the heights of the steps in $\rho_{*}(x)$, respectively. We choose $C_{1}$ to satisfy the normalization condition

$$
-\sum_{k=1} C_{k} X_{k}=\int d x \rho_{*}(x)=1 .
$$

If there exists such $k_{p}$ that satisfies $f^{k} p(0)=0$ and $f^{k}(0)$ $\neq 0$ for $\forall k<k_{p}$, the sum over $k$ is taken from 1 to $k_{p}$. Otherwise, the sum is taken from 1 to $\infty$.

Before treating the case of stochastic updating, let us analyze how the stationary state is affected by adding external force with infinitesimal amplitude $\delta$ for $p=1$ and $K=0$. Here we assume that the external force changes $x_{i}$ for every element by the given amount. Thus, when the force $\delta_{0}$ is applied at $t=0$, the distribution function at $t=1$ is expressed as

$$
\rho_{1}\left(x ; \delta_{0}\right)=\rho_{*}\left(x-\delta_{0}\right) .
$$

In the limit of $\delta_{0} \rightarrow 0$, the response of the mean field after $\tau$ steps is written as

$$
h_{\tau}=L_{\tau} \delta_{0},
$$

where $L_{\tau}$ is the linear coefficient for the response with delay of $\tau$ steps [12]. From Eq. (15), we calculate $L_{\tau}$ as follows:

$$
L_{\tau}=-\sum_{k=1}^{\infty} C_{k} X_{k+\tau}
$$

When the temporal series of the external force is given as $\left\{\delta_{t}\right\}$, the mean field $h_{t}$ is obtained as

$$
h_{t}=\sum_{\tau=1}^{\infty} L_{\tau} \delta_{t-\tau}
$$

within the linear approximation. Introducing a cutoff $d$, the state at the time $t$ can be described approximately by $d$-dimensional vector

$$
\boldsymbol{v}_{t} \equiv\left(\delta_{t-1}, \delta_{t-2}, \delta_{t-3}, \ldots, \delta_{t-d}\right) .
$$

In order to obtain the stability condition accurately, we must take the limit of $d \rightarrow \infty$. When the components of $\boldsymbol{v}_{t}$ is denoted as $v_{t}^{i}$, we obtain

$$
h_{t}=\sum_{i=1}^{d} L_{i} v_{t}^{i}
$$

The next step is to consider the case of $p=1$ and $K \neq 0$. In this case, the mean-field coupling yields the feedback force. The influence of the feedback force is described as

$$
v_{t+1}^{1}=K h_{t} .
$$

Taking Eq. (22) into account, the evolution of $v_{t}^{i}$ is described as

$$
v_{t+1}^{i}=\sum_{j=1}^{d} J_{i j} v_{t}^{j}
$$

where the matrix $J_{i j}$ is given by

$$
J_{i j}=\left(\begin{array}{cccccc}
K L_{1} & K L_{2} & K L_{3} & \cdots & K L_{\tau_{c}-1} & K L_{\tau_{c}} \\
1 & 0 & 0 & \cdots & 0 & 0 \\
0 & 1 & 0 & \cdots & 0 & 0 \\
\vdots & \vdots & \vdots & \ddots & \vdots & \vdots \\
0 & 0 & 0 & \cdots & 0 & 0 \\
0 & 0 & 0 & \ldots & 1 & 0
\end{array}\right) .
$$

The characteristic equation of the matrix (25) is given as

$$
\lambda^{d}=K \sum_{i=1}^{d} L_{i} \lambda^{d-i}
$$

which coincides with the results of Refs. [12,24]. The roots of Eq. (26) are denoted as $\mu_{i}$. In the case of the synchronous updating $(p=1)$, if all $\mu_{i}$ lie within the unit circle in the complex plane, the stationary state is stable [24].

Let us now return to the asynchronous updating case ( $p$ $<1)$. Here we define the vector $\boldsymbol{v}_{t}$ to satisfy Eq. (22). Thus, $v_{t}^{i}$ represents the contribution from the past perturbations through $i$ times of updating. Taking into account that the elements are updated with probability $p$ and fixed with probability $1-p$, we obtain

$$
v_{t+1}^{i}=\sum_{j=1}^{d}\left[p J_{i j}+(1-p) \delta_{i j}\right] v_{t}^{j} .
$$

The characteristic equation for Eq. (27) is given as

$$
\left(\frac{\lambda-1+p}{p}\right)^{d}=K \sum_{i=1}^{d} L_{i}\left(\frac{\lambda-1+p}{p}\right)^{d-i}
$$

Comparing Eq. (28) with Eq. (26), $\lambda$ in Eq. (26) is replaced with $(\lambda-1+p) / p$ in Eq. (28), and thus the stability condition for Eq. (27) becomes

$$
\left|1-p+p \mu_{i}\right|<1 \quad(\text { for all } i)
$$

This condition means all $\mu_{i}$ lie within the circle with center $(1-1 / p, 0)$ and radius $1 / p$ in the complex plane (Fig. 4). In the limit $p \rightarrow 0$, the condition $(29)$ becomes $\operatorname{Re}\left(\mu_{i}\right)<1$. Consequently, if all $\mu_{i}$ satisfy $\operatorname{Re}\left(\mu_{i}\right)<1$, the system has the threshold $p_{c}$.

For example, we investigate a simple case $a=(1$ $+\sqrt{5}) / 2$, where $f^{3}(0)=0$. Thus, $k_{p}$ is 3 and $X_{k}$ is periodic. In this case, the characteristic equation (26) can be solved 


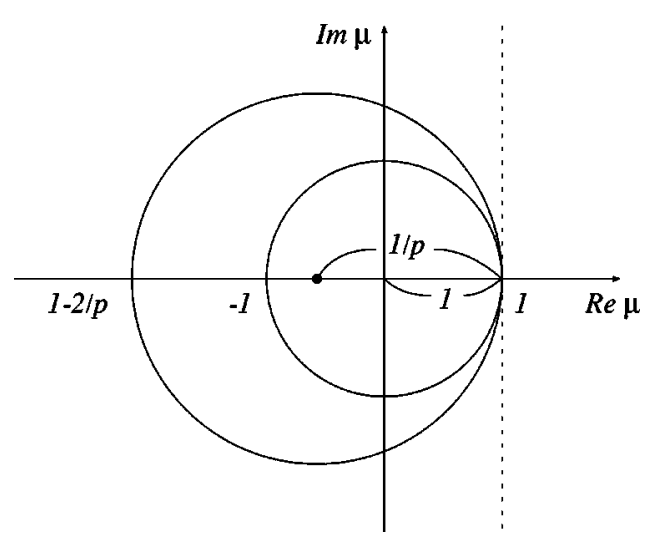

FIG. 4. The stability condition. If all solutions $\mu_{i}$ of Eq. (26) lie within the large (unit) circle, the stationary state is stable for $p$ $<1(p=1)$. Thus, all $\mu_{i}$ lie on the left of the dotted line, there exists threshold value $p_{c}$.

analytically in the limit of $d \rightarrow \infty$. From Eqs. (15) and (19), the linear coefficient $\left\{L_{t}\right\}$ is given as

$$
\begin{gathered}
L_{3 n+1}=L_{1}=-\frac{5+\sqrt{5}}{10}, \\
L_{3 n+2}=L_{2}=-\frac{5-\sqrt{5}}{10}, \\
L_{3 n+3}=L_{3}=1 .
\end{gathered}
$$

Let us assume that $\mu$ satisfies condition $|\mu|>1$. Then we rewrite the characteristic equation (26) in the limit $d \rightarrow \infty$ as follows:

$$
1=K \sum_{i=1}^{\infty} L_{i} \mu^{-i}=K \frac{L_{1} \mu^{-1}+L_{2} \mu^{-2}+L_{3} \mu^{-3}}{1-\mu^{-3}} .
$$

From this, $\mu$ is given as

$$
\mu=\frac{K L_{1}-1 \pm \sqrt{\left(K L_{1}-1\right)^{2}-4 K-4}}{2} .
$$

When $(45-21 \sqrt{5}) / 2<K<(5+3 \sqrt{5}) / 2$, the part in the square root in Eq. (32) is negative and thus the solution (32) is complex conjugate pair. Then, we obtain $|\mu|=\sqrt{K+1}$. Thus, $|\mu|>1$ holds for $0<K<(5+3 \sqrt{5}) / 2$. In this case, the threshold $p_{c}$ is given by

$$
p_{c}=\frac{K L_{1}-3}{K L_{1}-3-K}
$$

Figure 5 shows the correspondence between the above estimation for $p_{c}$ and the numerical results. It indicates the good agreement, except for the lower right corner. The estimation implies $p_{c}$ tends to 1 in the limit of $K \rightarrow 0$, and we think that the gap in the corner appeared because the amplitude of the collective motion for small $K$ is very small [estimated at $\exp (-C / K)$ for the case with $p=1[11,12]]$.

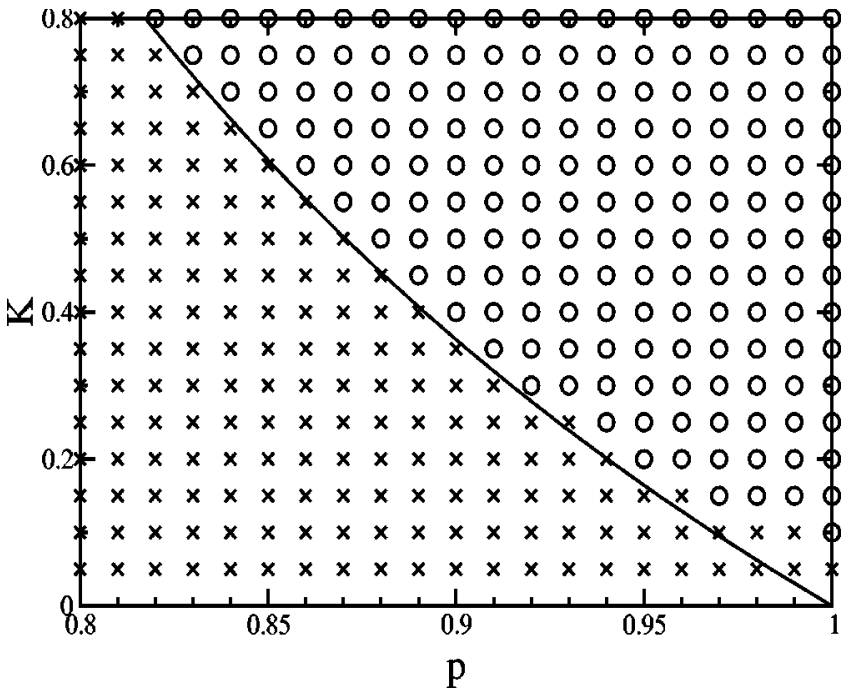

FIG. 5. Phase diagram of the collective motion for $a=(1$ $+\sqrt{5}) / 2$. The numerical results are obtained by calculation of Eq. (11) with $2^{18}$ grids. Circles and crosses represent the cases of $\left\langle h^{2}\right\rangle^{1 / 2}>10^{-12}$ and $\left\langle h^{2}\right\rangle^{1 / 2}<10^{-12}$, respectively. The solid line represents the theoretical prediction (33).

For such special values of $a$, where $\left\{X_{k}\right\}$ falls on a periodic orbit, we can solve the characteristic equation by the above method. In this case, the characteristic equation has no solution $\mu$ that satisfies $\operatorname{Re}(\mu)>1$ for adequately weak coupling. Thus there exists the threshold. For the general values of $a$, it is difficult to solve the characteristic equation. The stability condition is evaluated only approximately with a large but finite value of the cutoff $d$. The calculation for several values of $a$ indicates that the threshold value $p_{c}$ approaches to 1 for $K \rightarrow 0$, as is seen in Fig. 5 for $a=(1$ $+\sqrt{5}) / 2$.

\section{SUMMARY AND REMARKS}

This study has explored the globally coupled tent maps with stochastic updating. We introduced the updating rate $p$ and examined how the collective behavior changes as $p$ varies. In the case of $a<1$, the collective motion has a sequence of bifurcations, which is similar to the period doubling cascade. In the case of $a>1$, the amplitude of the collective motion decreases as $p$ decreases. For the both cases, we observed the threshold $p_{c}$, below which the collective motion vanishes. We estimated successfully the threshold $p_{c}$ by the linear stability analysis of the stationary state.

For weak coupling, the threshold $p_{c}$ is likely to remain near 1 . Thus, a tiny asynchronousnism may extinguish the collective motion. Therefore, when GCM is used as model of real systems, we keep in mind that even if the updating rule is almost synchronous, the effect of asynchronous updating should not be ignored.

\section{ACKNOWLEDGMENT}

This research was supported partly by the Japan Society of Promotion of Science under the Contract No. RFTF96I00102. 
[1] K. Kaneko, Physica D 41, 137 (1990).

[2] K. Kaneko, Phys. Rev. Lett. 65, 1391 (1990); Physica D 55, 368 (1992).

[3] H. Chaté and P. Manneville, Europhys. Lett. 17, 409 (1991); 17, 291 (1992); Prog. Theor. Phys. 87, 1 (1992).

[4] N. Nakagawa and Y. Kuramoto, Prog. Theor. Phys. 89, 313 (1993).

[5] A.S. Pikovsky and J. Kurths, Phys. Rev. Lett. 72, 1644 (1994); Physica D 76, 411 (1994).

[6] K. Kaneko, Physica D 86, 158 (1995).

[7] W. Just, J. Stat. Phys. 79, 429 (1995); Physica D 81, 317 (1995).

[8] S.V. Ershov and A.B. Potapov, Physica D 86, 532 (1995); 106, 9 (1997).

[9] S. Morita, Phys. Lett. A 211, 258 (1996).

[10] S. Morita, Phys. Lett. A 226, 172 (1997).

[11] N. Nakagawa and T.S. Komatsu, Phys. Rev. E 57, 1570 (1998); 59, 1675 (1998).

[12] T. Chawanya and S. Morita, Physica D 116, 44 (1998).

[13] T. Shibata, T. Chawanya, and K. Kaneko, Phys. Rev. Lett. 82,
4424 (1999).

[14] T. Shibata and K. Kaneko, Physica D 124, 177 (1998).

[15] G. Pérez and H.A. Cerdeira, Phys. Rev. A 46, 7492 (1992).

[16] G. Pérez, S. Sinha, and H.A. Cerdeira, Physica D 63, 341 (1993).

[17] G. Pérez, S. Sinha, and H.A. Cerdeira, Phys. Rev. E 54, 6936 (1996).

[18] J. Rolf, T. Bohr, and M.H. Jensen, Phys. Rev. E 57, R2503 (1998).

[19] G. Abramson and D.H. Zanette, Phys. Rev. E 58, 4454 (1998).

[20] S. Sinha, Phys. Rev. E 57, 4041 (1998).

[21] H.J. Blok and B. Bergersen, Phys. Rev. E 59, 3876 (1999).

[22] Y. Jiang, A. Antillón, and J. Escalona, Phys. Lett. A 262, 403 (1999).

[23] P.-M. Binder and D.H. Campos, Phys. Rev. E 53, R4259 (1996).

[24] G. Keller, Prog. Prob. 46, 183 (2000).

[25] For $p=0$, all elements are never updated. Therefore, when we consider the limit of $p \rightarrow+0$, the time $t$ must be rescaled by $p$ to keep the motions. 